Proceedings of the International Conference on Oxide Materials for Electronic Engineering, May 29-June 2, 2017, Lviv

\title{
Image Formation by Ink-Jet Printing in Micro- and Nanoporous Anodic Alumina Film with Capsulating
}

\author{
B. Kazarkin, Y. Mukha, A. Stsiapanau and A. Smirnov \\ Belarusian State University of Informatics and Radioelectronics, Minsk, Belarus
}

\begin{abstract}
The image formation process based on the method of ink-jet printing in films of anodic aluminum oxide has been developed and investigated. The image resolution of $500 \mathrm{dpi}$ was achieved on the films with $150 \mu \mathrm{m}$ depth of pores. The films of anodic aluminum oxide were produced on aluminum foil by partial or through anodizing of aluminum followed by filling the pores with ink and their capsulation for protection. The obtained films have exceptional thermal and chemical stability. The low-temperature and low-cost process described in this article is promising for mass production of elements of microelectronic devices.
\end{abstract}

DOI: 10.12693/APhysPolA.133.771

PACS/topics: nanostructured alumina oxide, ink-jet printing, ink capsulation

\section{Introduction}

The tendency to reduce the cost of micro- and optical electronic devices, traditionally solved by increasing the size of processed substrates, now faces a number of physical limitations. An alternative is "roll-to-roll" technology using flexible metal, glass, or polymer substrates [1]. The main disadvantage of this technology is insufficient surface quality and weak protective properties of flexible substrates. One of the effective methods for solving this problem is the use of special protective layers which enables to protect functional materials applied on flexible substrates from the negative influence of both the substrate and the environment.

In this paper we investigated the technology of image formation by the method of ink-jet printing onto micro- and nanoporous alumina oxide. Following closing of pores (capsulation) allows to protect the ink from external influences.

We investigated thermal and chemical stability of ink in nanostructured porous anodic aluminum oxide (PAO) matrices with a vertical pore orientation, as well as the ability to produce high spatial resolution of images formed by ink-jet printing. It should be noted that the processes of aluminum deposition and the formation of porous anodic oxide are low-temperature processes and are fully compatible with "roll-to-roll" technologies, including those on flexible polymer substrates.

\section{Experimental part}

There are many designs of electrochemical cells that are currently used for anodizing processes. Each of them has its advantages and disadvantages. We used electrochemical cells, shown in Fig. 1. This cell has a U-shaped cathode and is equipped with a sample holder with a passivated dense anodic oxide. The holder, due to the strong clamping, provides a point contact isolated from the electrolyte. This cell allows anodization of both sides of the sample simultaneously, and the U-shaped cathode

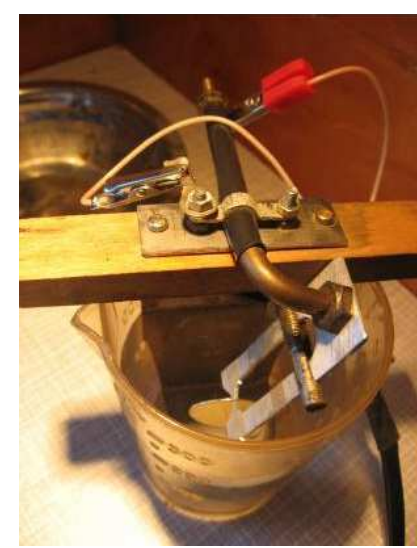

Fig. 1. Electrochemical cell with a U-shaped cathode.

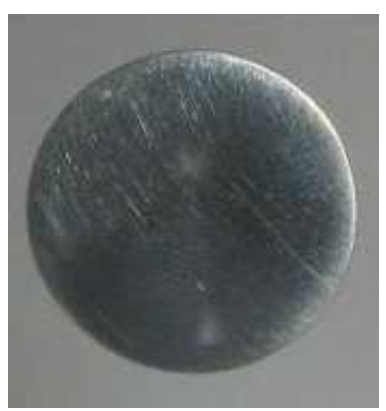

Fig. 2. Sample.

ensures the uniformity of the properties of the resulting structure across the entire anodization surface.

Aluminum samples (Fig. 2) contain not less than $99.55 \% \mathrm{Al}$ and $0.45 \%$ impurities of silicon, manganese, and other impurities and are shaped as a circle with a diameter of $12 \mathrm{~mm}$ and thickness of $400 \mu \mathrm{m}$. The surface of the samples was treated to a mirror finish by doublesided mechanical polishing. Then it was washed in an ultrasonic bath CT-400D for 5 min. 
The anodization parameters were recorded using a digital multimeter MAS-345 connected to a personal computer, which allowed for automation of recording the time dependences of the voltage $U(t)$ and current $I(t)$. The pore size and porosity were determined using a scanning electron microscope SUPRA-55WDS.

Sulfuric acid $\mathrm{H}_{2} \mathrm{SO}_{4}$ (15\%) and $0.25 \mathrm{M}$ orthophosphoric acid $\mathrm{H}_{3} \mathrm{PO}_{4}$ were used for the experiments. This choice of chemical reagents can be explained by the need to obtain pores with different diameters. For sulfuric acid, the diameter is $10-20 \mathrm{~nm}$, and $120-140 \mathrm{~nm}$ for orthophosphoric acid (Fig. 3) [2, 3].

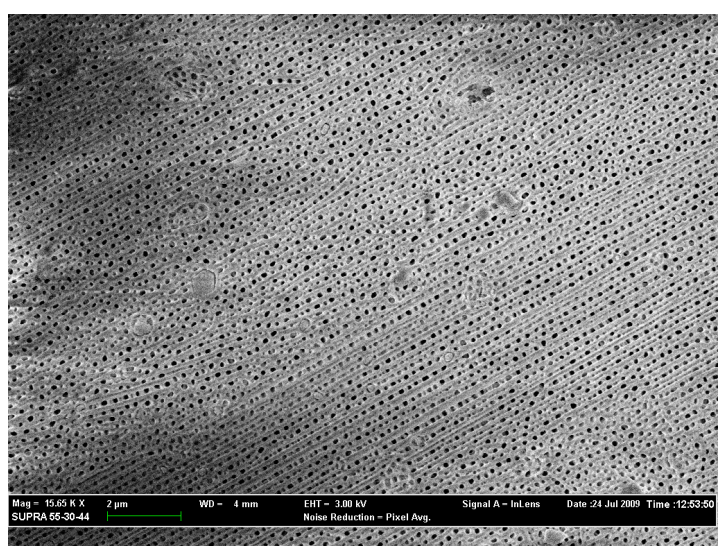

Fig. 3. SEM image of structure of the PAO.

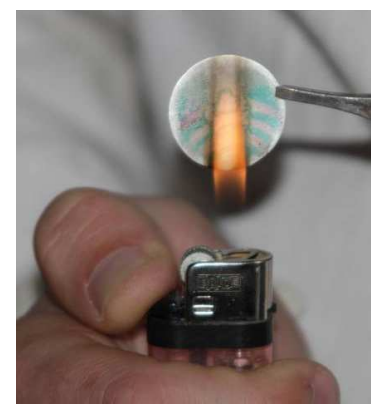

Fig. 4. Testing the sample for thermal resistance.

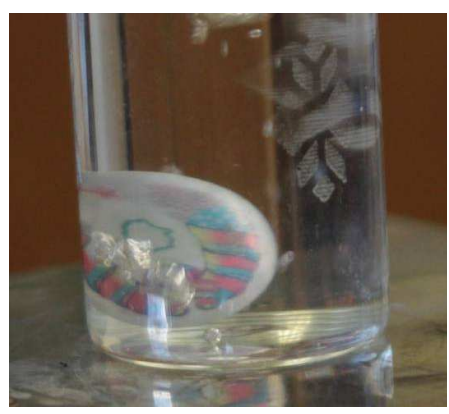

Fig. 5. Testing the specimen for chemical resistance.

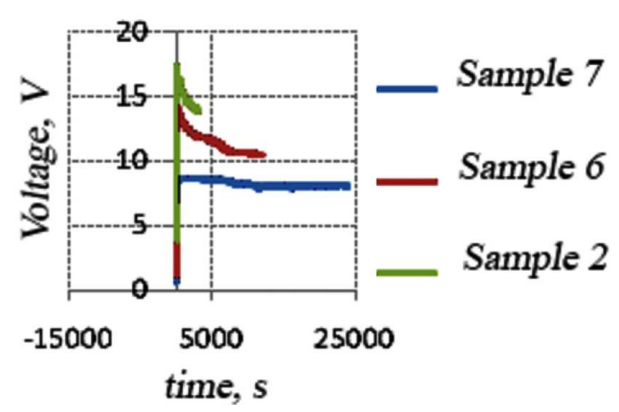

Fig. 6. Dependence of the anodizing voltage on the anodizing time.

In the course of the experiments, we studied the processes of formation and filling of pores with ink depending on the diameter and depth of pores. Boiling water and an available heat source (1minute exposure to flame (a lighter) with temperature of $700^{\circ} \mathrm{C}$ ) were used to study the stability of the obtained capsulated ink under thermal (Fig. 4) and chemical influences (Fig. 5). Their mechanical resistance was determined by bending deformation in opposite directions, as well as by polishing the samples i.e. attempting to clear off the image.

Preliminary to applying the pattern, it is necessary to ensure the drying of the samples. For this purpose, we have designed a special device that allows for uniform temperature maintenance within the range of $30-90^{\circ} \mathrm{C}$ across the area of the sample. As a rule, drying of the samples was carried out at a temperature of $50^{\circ} \mathrm{C}$, at which moisture evaporated from the pores, but the pores themselves did not close.

The subsequent filling of pores with ink was carried out on the HP Photosmart D5363 printer with resolution of 500 dpi.

\section{Results and discussion}

The anodizing process was carried out in the constant current mode. This choice is explained by the need to accurately count the amount of anodization current, which plays a key role in determining the depth of pores of the produced porous alumina films [4].

Samples obtained in solutions of sulfuric acid have pores with diameter of $10-20 \mathrm{~nm}$ and color ink particles easily penetrate them. It is experimentally established that filling in the black ink gave a blurred image even in solutions based on phosphoric acid which were used to form structures with pore diameters of 120-140 nm. It can be explained by the fact that the particle size of the black ink is much larger than that of the other inks. The use of color inks for printing on samples prepared in solutions containing orthophosphoric acid had the following drawback: immediately after printing, a pattern of excellent quality was observed in the pores, however, because of the large pore diameters, part of the ink leaked off the pores prior to their capsulation. Therefore, it was impossible to obtain an image of the desired quality. As a 
TABLE I

Profiles of anodizing processes. Time $[\mathrm{s}]-\tau$, current density $\left[\mathrm{mA} / \mathrm{cm}^{2}\right]-\rho$, transmitted current $\left[\mathrm{A} \mathrm{s} / \mathrm{cm}^{2}\right]-j$.

\begin{tabular}{|c|c|c|c|c|}
\hline \multirow[b]{2}{*}{ sampl } & 1 & 2 & 3 & 4 \\
\hline & & & & \\
\hline$\tau$ & 6000 & 3000 & 15000 & 3000 \\
\hline$\rho$ & 15 & 30 & 7.5 & 15 \\
\hline$j$ & 90 & 90 & 112.5 & 45 \\
\hline \multirow[b]{2}{*}{ samp } & 5 & 6 & 7 & 8 \\
\hline & & & & \\
\hline$\tau$ & 6000 & 12000 & 24000 & 1500 \\
\hline$\rho$ & 30 & 15 & 7.5 & 30 \\
\hline$j$ & 180 & 225 & 180 & 45 \\
\hline
\end{tabular}

result, color ink and a solution containing sulfuric acid were chosen for further research.

Anodizing process modes are presented in Table I. The solution contained $15 \%$ sulfuric acid $\mathrm{H}_{2} \mathrm{SO}_{4}$.

Figure 6 shows the dependence of voltage on time for three samples with different current densities $\rho$ : 30, 15, and $7.5 \mathrm{~mA} / \mathrm{cm}^{2}$, respectively. In cases where the value of the transmitted current is the same, the depth of the pores will be greater in those samples, the anodization time of which is longer. Table I shows that the samples 3,6 , and 7 , i.e. with the greatest anodizing time, have the best color rendition. The pore depth of these samples was about $150 \mu \mathrm{m}$.

The image quality was evaluated visually, before and after the pore was sealed, using a Canon EOS 500D digital camera of high resolution and an optical microscope. As we can see in Table I below, samples of excellent quality were produced using sulfuric acid. They are hardly different from images printed on paper. Samples 4 and 8 turned out to be blurred. This is explained by the small transmitted current ( $45 \mathrm{~A} \mathrm{~s} / \mathrm{cm}^{2}$ ), which resulted in small depth of the pores and consequently led to ink overfill.

It is necessary to note the importance of observing the same climatic conditions (humidity and air temperature) of the experiments. We could observe the Kelvin law in action. At high air humidity, the pores were filled with condensed water, which make further printing of such a sample extremely difficult, since a longer drying time with less moisture was required.
Pore sealing (capsulation) occurs during the chemical interaction of $\mathrm{Al}_{2} \mathrm{O}_{3}$ and $\mathrm{H}_{2} \mathrm{O}$ which produces insoluble compound $\mathrm{Al}(\mathrm{OH})_{3}$ when distilled water interacts with a film of porous anodic aluminum oxide. The process temperature is $90-95^{\circ} \mathrm{C}$, the duration time is $10 \mathrm{~min}$.

We observed exceptional resistance of samples to mechanical, thermal (Fig. 4) and chemical exposure (Fig. 5). If a sample is exposed to mechanical deformation, open fire, or boiling water, the image quality will not change. Thus, the developed technology can be used to apply images to metal surfaces that are operated under extreme conditions (car engines, military tags, etc.). It can also serve as a protective structure for a functional material (luminescent material, quantum dots, etc.) filled into micro- and nanoporous aluminum oxide.

\section{Conclusion}

The obtained results demonstrate the conceptual possibility of image formation in an anodic aluminum oxide matrix by filling the pores with ink using the method of ink-jet printing. Sealing micro- and nanopores pores enables to obtain capsulated materials in a protective layer from $\mathrm{Al}_{2} \mathrm{O}_{3}$ matrix. Such materials are resistant to mechanical, physical and chemical effects. Applying various filling materials (e.g. luminescent material for light emission) it is possible to produce functional layers.

\section{Acknowledgments}

The work is partly supported by Belarusian Republican Foundation for Basic Researches (grant No. T16P200).

\section{References}

[1] E. Schwartz, Roll to Roll Processing for Flexible Electronics, Cornell University, 2006.

[2] A. Hubarevich, M. Marus, A. Stsiapanau, A. Smirnov, J. Zhao, W. Fan, H. Wang, X.W. Sun, Phys. Status Solidi A 212, 2174 (2015).

[3] M. Marus, A. Hubarevich, H. Wang, Y. Mukha, A. Smirnov, H. Huang, X.W. Sun, W. Fan, Opt. Mater. Expr. 6, 2655 (2016).

[4] A. Stsiapanau, P. Jaguiro, A. Smirnov, H. Kwok, A. Murauski, Y. Jacob, Nanostructured metal transparent conductive layer and method of its self-ordered fabrication from valve metal film, Patent application No. $61 / 213,283$ on $05 / 26 / 2009$, USA. 